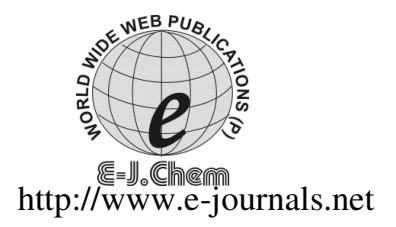

ISSN: 0973-4945; CODEN ECJHAO

E-Journal of Chemistry

2011, 8(4), 1686-1695

\title{
Effect of Thickness on Structural and Optical Properties of Tetra-tert- butyl-2, 3-naphthalocyanine Thin Films
}

\author{
I. DHANYA* and C S MENON \\ School of Pure and Applied Physics, Mahatma Gandhi University \\ Priyadarshini Hills, Kottayam, Kerala, 686 560, India \\ dhanya.iv@gmail.com
}

Received 19 February 2011; Accepted 16 April 2011

\begin{abstract}
Amorphous tetra-tert-butyl-2, 3-naphthalocyanine thin films have been deposited using physical vapor deposition technique under a varied thickness by adjusting the coating time. By analyzing the $\mathrm{x}$-ray diffraction, the structure of as deposited films is found to be non-crystalline. Different optical properties of these thin films have been investigated by means of optical absorption and reflection spectra. Various optical constants like band gap energy, $\mathrm{E}_{\mathrm{g}}$ the width of band tails of localized states into the gap, $\mathrm{E}_{\mathrm{U}}$ and steepness parameter, $\beta$ gets calculated and the variation of different optical parameters like refractive index, extinction coefficient, dielectric constants, optical conductivity and surface and volume energy losses with photon energy are estimated.
\end{abstract}

Keywords: Naphthalocyanines, Non-crystalline, Bandgap energy, Optical constants, Optical conductivity

\section{Introduction}

More complex options to porphyrines, naphthalocyanines have been extensively used as potential materials for optoelectronic and electronic devices for solar cells $\mathrm{s}^{1-3}$. Tert-butyl substituted naphthalocyanine, as one of phthalocyanine derivatives is known for frequency and time domain spectral hole burning optical storage devices ${ }^{4}$ due to the presence of large inhomogeneous broadening, relatively narrow holes at low temperatures and a built in proton photo transformation mechanism between different tautomers. Several free based naphthalocyanine derivatives exhibit a strong O-O absorption band region in the region $800 \mathrm{~nm}$ matching the wavelength of most semiconductor diode lasers and Ti-Sapphire lasers. Most studies related to phthalocyanine derivatives are addressed to the structural aspects of the molecular arrangement in thin films and in solution phase using UPS, XPS or NEXAFS ${ }^{5-8}$. 
There are little studies on determination of various optical constants of naphthalocyanine compounds especially in thin film configuration even though some optical studies exist ${ }^{9,10}$. Here we are intended to prepare 2,11,20,29-tetra-tert-butyl2,3-naphthalocyanine (TTBNc) thin films and monitored the x-ray diffraction pattern, absorption and luminescence spectra. TTBNc is absolutely a metal free porphyrine with the ligands are not co-ordinate to any central metal ion, the electronic states are exclusively of porhyrazine ligand origin.

Due to high fluorescence quantum yield (0.01-0.4) of metal free TTBNc with respect to their metal centered compounds ${ }^{11}$ and a strong fluorescence spectrum is observed in these compounds especially in thin film states. Here thin film samples of TTBNc with varied thickness are investigated.

\section{Experimental}

The raw material for thin film sample preparation is 2,11,20,29-tetra-tert-butyl2, 3-naphthalocyanine (TTBNc) powder, procured from sigma -aldrich chemicals, USA. TTBNc thin films were deposited onto thoroughly cleaned micro glass substrate at room temperature, at a base pressure of $1 \times 10^{-5}$ torr using a Hind Hivac 12A4 coating unit. The evaporation was carried out by resistive heating of TTBNc powder from a molybdenum boat kept at a distance of $7 \mathrm{~cm}$ from the substrate by means of respective copper electrodes. Two sets of thin films are obtained on the same pressure condition by successive sets of coating. The thickness of the films is measured using thickness profilometer and is cross checked by tolansky's method ${ }^{12}$. The thicknesses are $825 \mathrm{~nm}$ and $195 \mathrm{~nm}$ for two sets of samples under investigation by varying their time of evaporation as 10 minutes and 2 minutes respectively with a variation of $\pm 15 \mathrm{~nm}$. Thin film nature of these samples has been well established through a series of spectroscopic methods including x-ray diffractogram, UV-Visible spectrum and photoluminescence techniques and there by various optical constants gets calculated.

The structure properties are investigated by means of an x-ray diffractometer PAN analytical (X'pert Pro) with $\mathrm{Cu} \mathrm{K} \mathrm{K}_{\alpha}$ radiations $\left(\lambda=1.5405 \mathrm{~A}^{0}\right)$ in the range of $2 \theta$ between $10^{\circ}$ and $40^{\circ}$. The optical measurements of the films like absorbance A, reflectance $\mathrm{R}$ of the films are carried out at room temperature using a Shimadzu UV-VIS 2450 spectrophotometer in the wavelength range from 190 to $900 \mathrm{~nm}$. The photoluminescence spectrum is taken using Horiba Jobin Yvon Flurolog 3 modular spetroflurometer with 450 watt Xenon lamp and having a Hanafpu R 928-28 photomultiplier tube.

\section{Results and Discussion}

\section{Structural characterization}

The crystal structure of the TTBNc thin films under different thickness is investigated by x-ray diffraction (XRD) patterns. Figure 1 shows the diffraction patterns of as deposited TTBNc thin films of varied thickness and are found to be almost non crystalline in nature.

\section{Optical band gap and Urbach energy}

The optical absorbance and reflectance of the films are shown in Figure 2. The maximum absorbance is corresponding to B band for the two films with a varying wave length of $310 \mathrm{~nm}$ for $825 \mathrm{~nm}$ thick film and $340 \mathrm{~nm}$ for $195 \mathrm{~nm}$ thick film. In the absorption spectra the Q bands having a splitting of Qx and Qy and is prominent while thickness increases. 


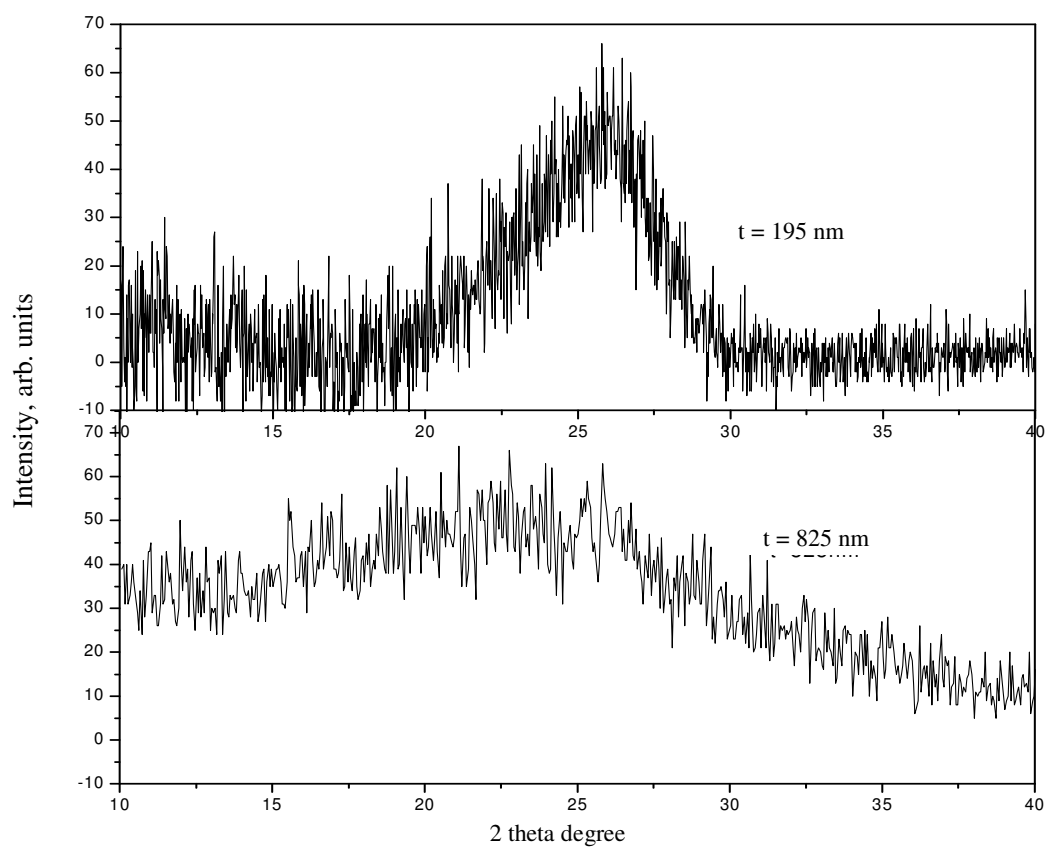

Figure 1. X-ray diffraction patterns of different TTBNc thin films
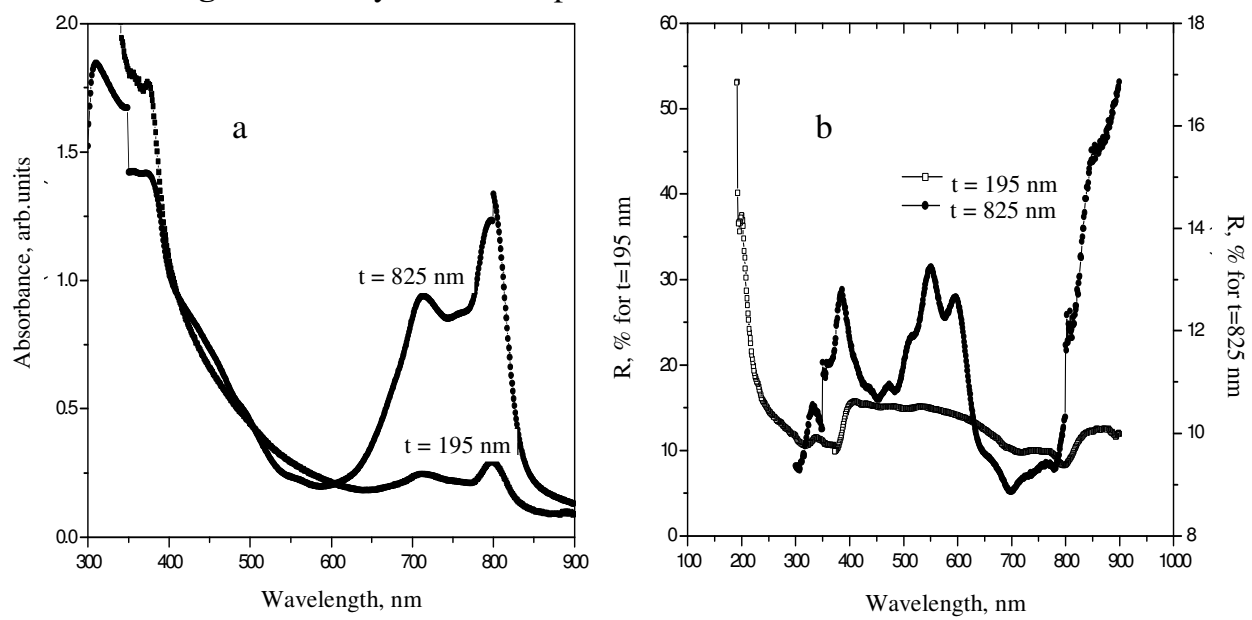

Figure 2. Optical absorbance (a) and reflectance (b) spectra of different TTBNc thin films From the absorbance spectra, the absorption coefficient, $\alpha$ and extinction coefficient, $\mathrm{k}$ are found out using the expressions ${ }^{13,14}$

$$
\alpha=\frac{2.303 \times A}{t}
$$

and

$$
k=\alpha \lambda / 4 \pi
$$

Where A, the absorbance; $t$, the thickness of thin films and $\lambda$ is the wavelength range. Graphs of $\alpha v s$. hv and $\mathrm{k} v s$. hv are plotted and shown in Figure 3. 

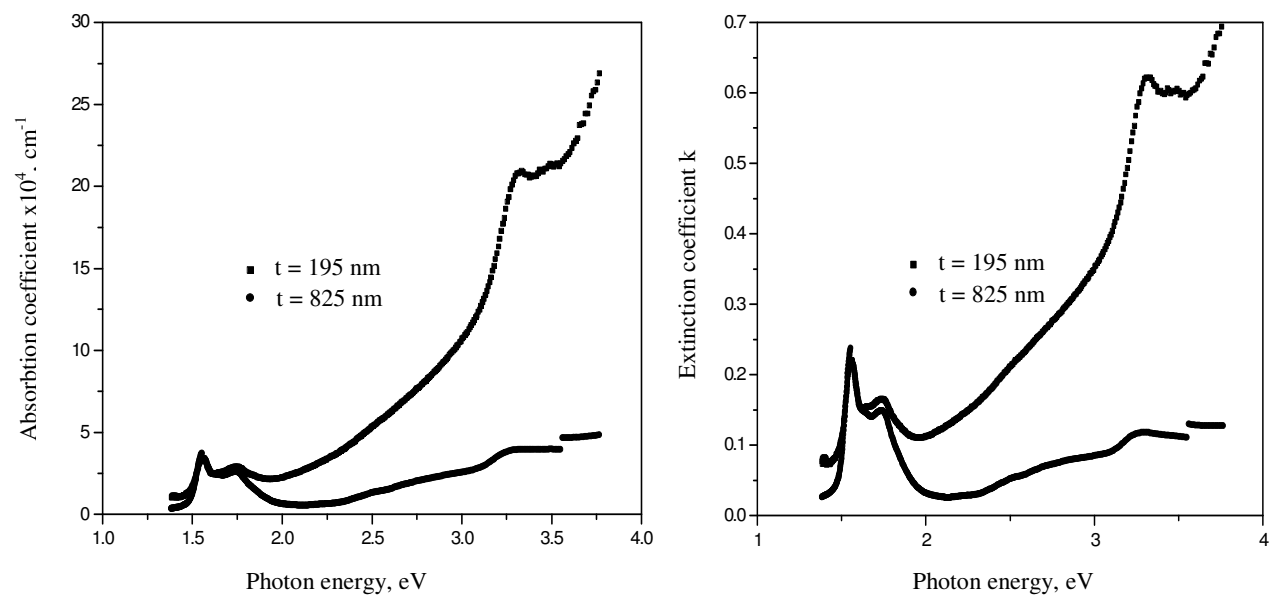

Figure 3. Absorption coefficient, $\alpha$ vs. photon energy and extinction coefficient, $\mathrm{k} v s$. photon energy for TTBNc thin films of different thicknesses

The optical band gap $\mathrm{E}_{\mathrm{g}}$ can be calculated from the following relationship ${ }^{15,16}$

$$
\alpha=\alpha_{0}\left(h v-E_{g}\right)^{n}
$$

Where $\mathrm{n}=1 / 2$ for direct allowed transition. Graph of $\alpha^{2} v s$. $\mathrm{h} v$ is plotted and is shown in Figure 4. Extrapolation of the plot to $\alpha^{2}=0$ determines the optical band gap. The band gap energy of TTBNc thin film is found to be $3.02 \mathrm{eV}$ and is invariant with thickness variation.

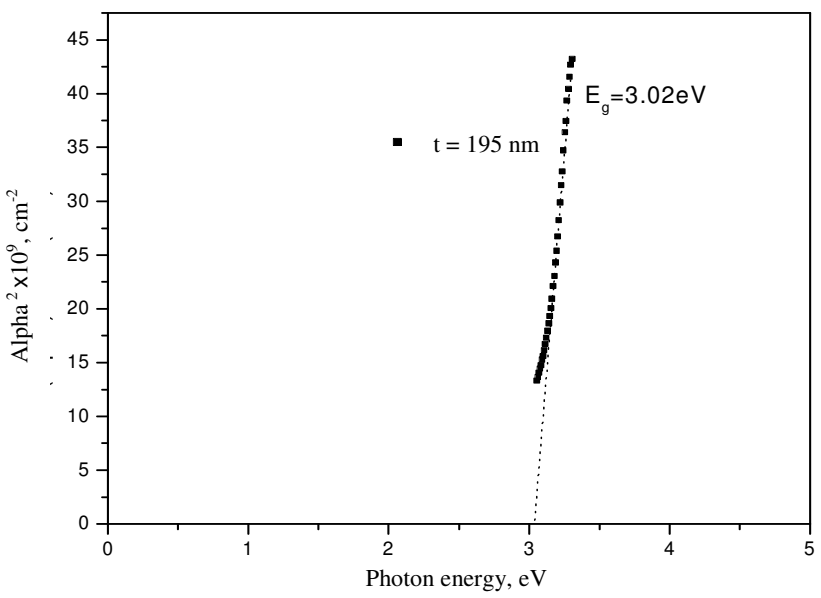

Figure 4. Typical plot of $\alpha^{2} v s$. hv of TTBNc thin film

The absorption coefficient near the fundamental absorption edge for as deposited TTBNc thin film is exponentially dependent on the incident photon energy and obeys the empirical Urbach relation, where ln $\alpha$ varies as a function of $h v$. The Urbach energy can be calculated by the following relation ${ }^{17}$

$$
\ln \alpha=\alpha_{0} \exp \left[\frac{h v-E_{I}}{E_{U}}\right]
$$


Where $\mathrm{E}_{\mathrm{I}}$ and $\alpha_{0}$ are constants and $\mathrm{E}_{\mathrm{U}}$ is the Urbach energy, which refers the width of the exponential absorption edge. Figure 5 represents the Urbach plot of TTBNc thin film and $\mathrm{E}_{\mathrm{U}}$ can be calculated from the slope of the graph. Urbach energy values of the films found to increase with increase in thickness due to the increase in the width of localized states in optical bands.

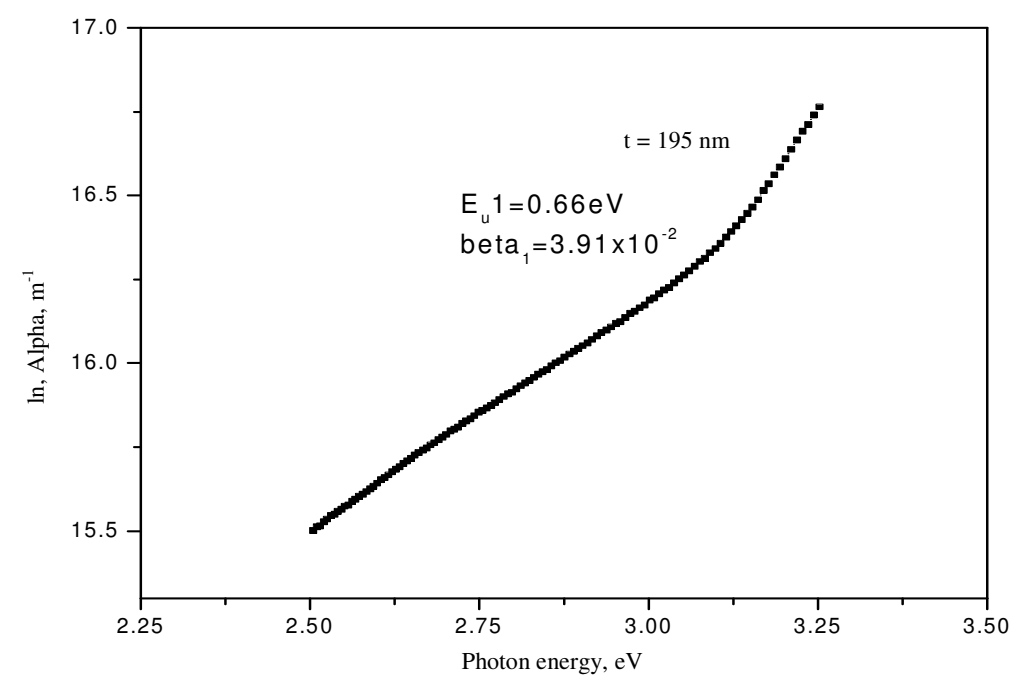

Figure 5. Typical Urbach plot of TTBNc thin film

From the Urbach plot, the steepness parameter $\beta$ can be empirically determined using the relation,

$$
\alpha=\alpha_{0} \exp \left[\frac{\beta}{K T}\left(E-E_{I}\right)\right]
$$

Where $\beta$, the steepness parameter, which characterizes the broadening of the absorption edge due to the electron- phonon interaction or exciton-phonon interaction ${ }^{18}$. From the above graph, the $\beta$ values suggest that the absorption edge changes with the variation in thickness.

\section{The refractive index and dielectric constants of TTBNc thin films}

In the optical parameters, dispersion plays an important role in the research for optical materials, because of its significance in optical communication and in designing devices for spectral dispersion. The reflectivity of an absorbing medium of indices $\mathrm{n}$ and $\mathrm{k}$ in air under normal incidence is given by ${ }^{19}$

$$
R=\frac{(n-1)^{2}+k^{2}}{(n+1)^{2}+k^{2}}
$$

and the optical properties of any solid material is characterized by the complex refractive index $\left(\mathrm{N}=\mathrm{n}\right.$-ik) and the complex dielectric constant $\left(\varepsilon=\varepsilon_{1}-i \varepsilon_{2}\right)$. Here the dispersion relates to the real part $\varepsilon_{1}$ and $\varepsilon_{2}$ is a measure of the dissipative rate of the wave in the medium. The real and imaginary part of dielectric constants are related to $\mathrm{n}$ and $\mathrm{k}$ values through the relations,

$$
\varepsilon_{1}=n^{2}-k^{2}
$$




$$
\varepsilon_{2}=2 n k
$$

Figure $6 \& 7$ represent the refractive index and $\varepsilon_{1}$, $\varepsilon_{2}$ dependence of films with their photon energy ${ }^{19}$. The maximum value of refractive index is found to be 2.35 and 2.27 for $825 \mathrm{~nm}$ and $195 \mathrm{~nm}$ thick films respectively with their $\varepsilon_{1}, \varepsilon_{2}$ variation are almost linear.

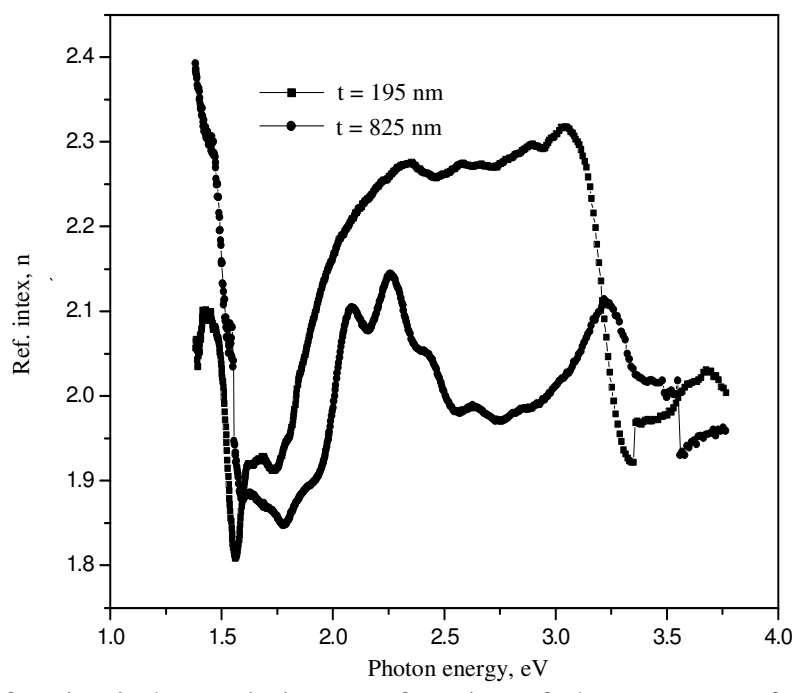

Figure 6. Refractive index variation as a function of photon energy for TTBNc thin films

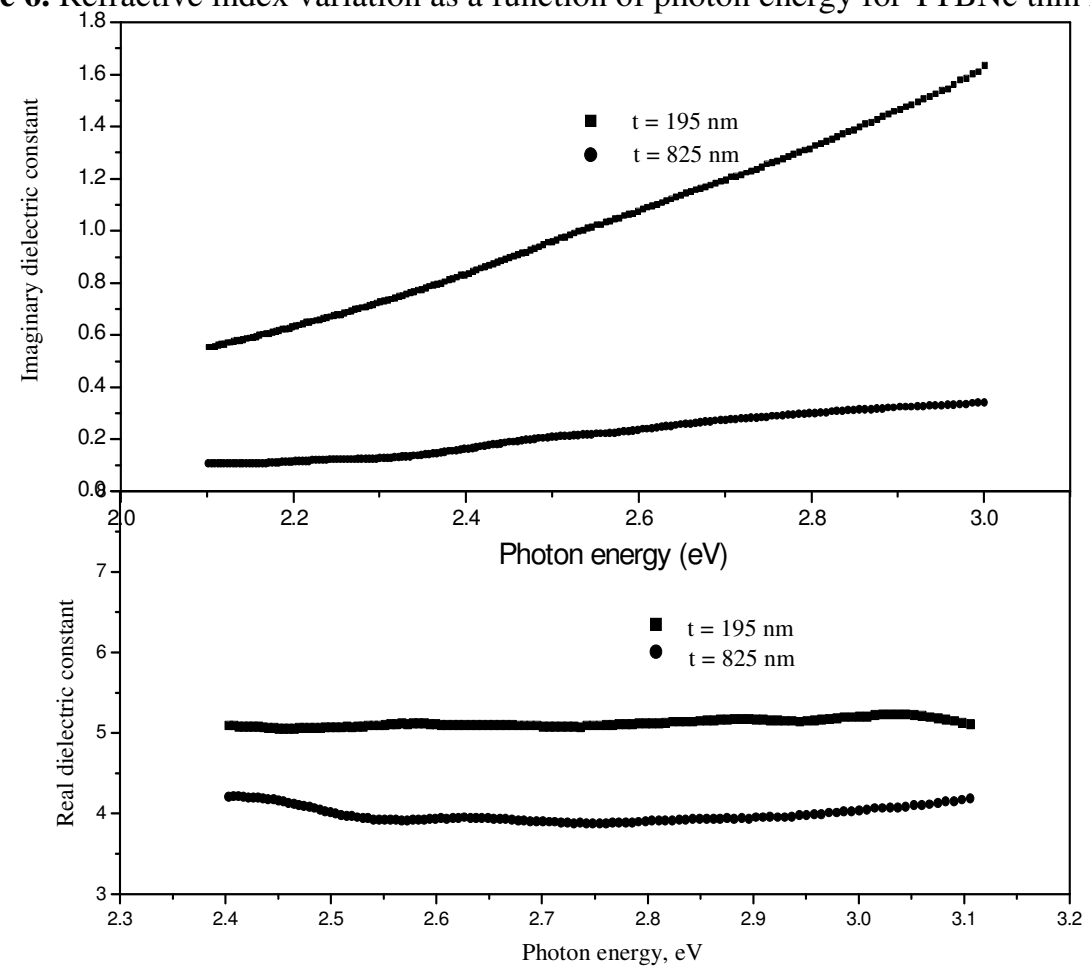

Figure 7. Variation of $\varepsilon_{1}$ and $\varepsilon_{2}$ as a function of photon energy for TTBNc thin films 


\section{Optical conductivity of TTBNc thin films}

The optical conductivity parameters, $\sigma 1$ and $\sigma 2$ are real and imaginary components respectively with their dependence on $\varepsilon_{1} \& \varepsilon_{2}$ can be described as ${ }^{20}$

$$
\sigma_{1}=\omega \varepsilon_{2} \varepsilon_{0} \text { and } \sigma_{2}=\omega \varepsilon_{1} \varepsilon_{0}
$$

Where $\omega$ is the angular frequency, $\varepsilon_{0}$ is the free space dielectric constant. The dependence of real $\sigma_{1}$ and imaginary $\sigma_{2}$ parts of optical conductivity with photon energy is as shown in Figure 8. The increase in photo conductivity due to the variation of photon energy is in a linear manner and is due to the excess electrons emitted by means of photon energy.

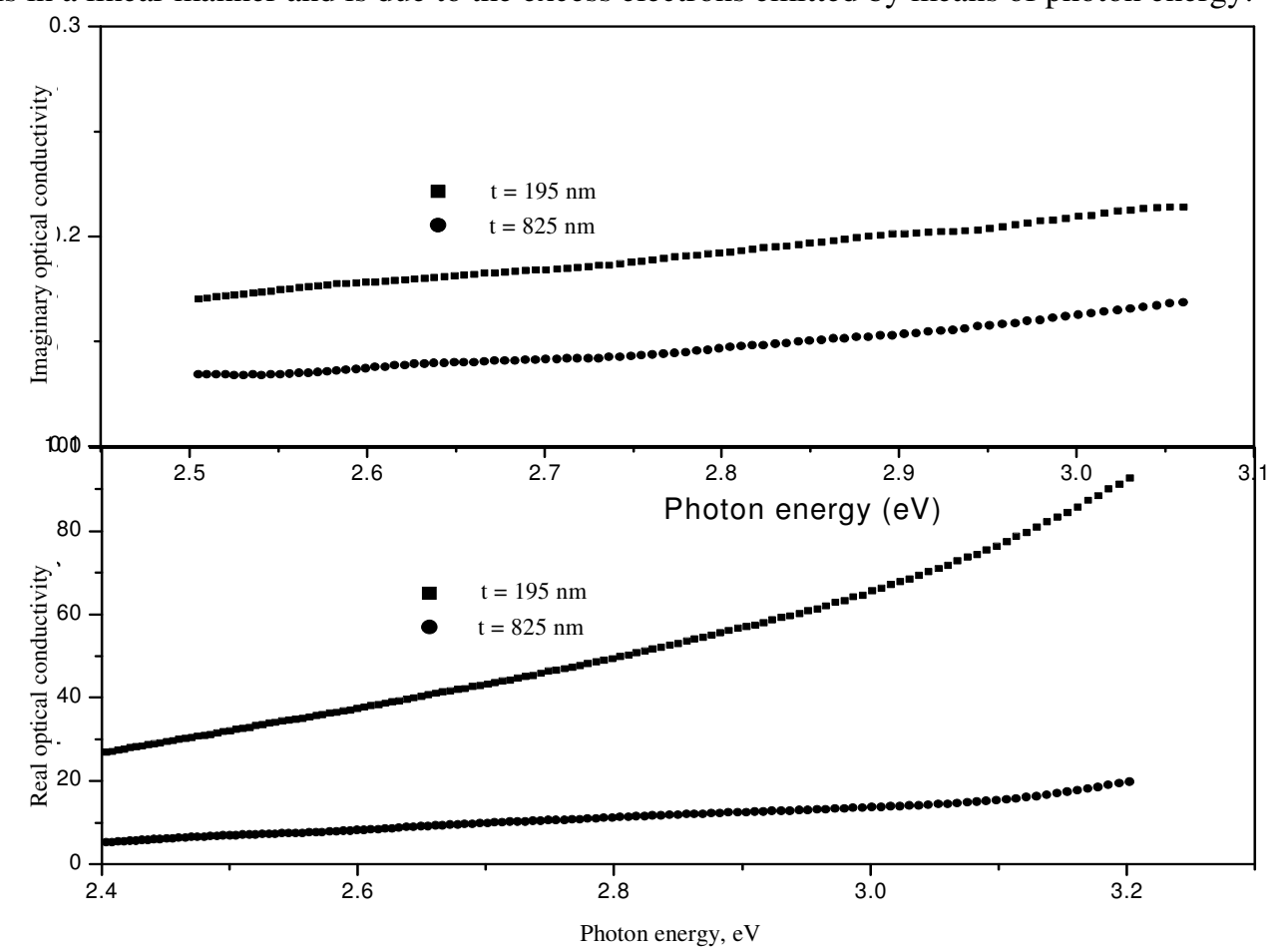

Figure 8. Real $\left(\sigma_{1}\right)$ and imaginary $\left(\sigma_{2}\right)$ part of different TTBNc thin films

Surface and volume energy loss functions of TTBNc thin films

The obtained results of optical conductivity can be further analyses through graphical representations of volume energy loss and surface energy loss as a function of photon energy (in Figure 9) to detect the existence of any possible allowed optical transition. Volume energy loss can be represented as ${ }^{21}$

$$
[-\operatorname{Im}(1 / \varepsilon)]=\varepsilon_{2} /\left(\varepsilon_{1}^{2}+\varepsilon_{2}^{2}\right)
$$

and the Surface energy loss

$$
[-\operatorname{Im}(1 /(\varepsilon+1))]=\varepsilon_{2} /\left[\left(\varepsilon_{1}+1\right)^{2}+\varepsilon_{2}^{2}\right]
$$



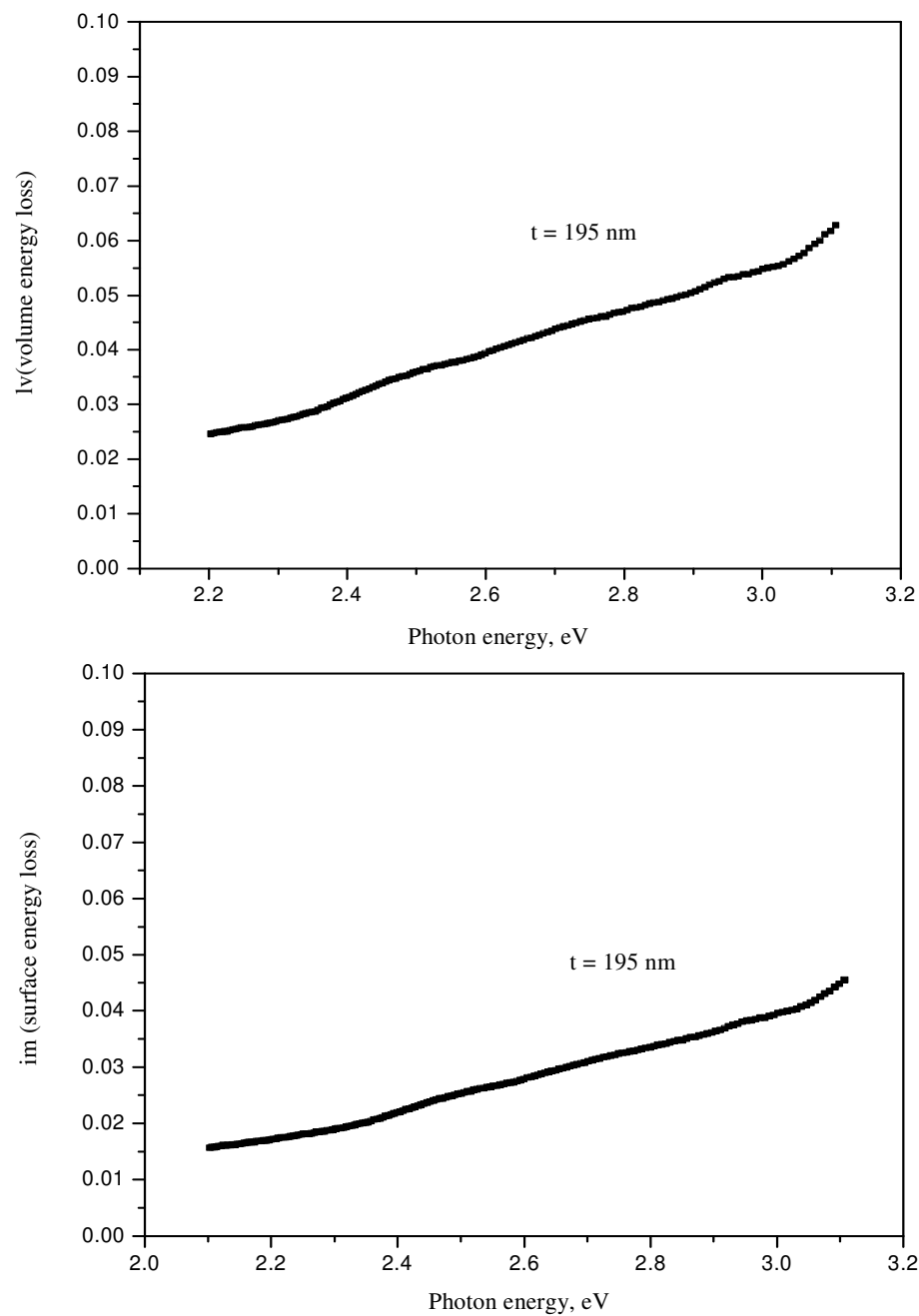

Figure 9. Typical (a) volume energy loss and (b) surface energy loss of TTBNc thin film as a function of photon energy

Both the two energy losses have a linear variation with the corresponding photon energy and found a maximum energy loss near the optical band gap energy. By increasing the amount of deposition, there is an effect of variation in optical constants and most of them in linear. This variation can be discussed in terms of defects present in the composition, due to the presence of localized states in the optical band gap. The different optical constants with respect to their thicknesses are listed in Table 1.

Table 1. Different optical constants of TTBNc thin films of varied thickness

\begin{tabular}{ccccc}
\hline $\begin{array}{c}\text { Thickness of film } \\
\pm 15 \mathrm{~nm}\end{array}$ & $\begin{array}{c}\mathrm{E}_{\mathrm{g}} \\
\pm 0.01(\mathrm{eV})\end{array}$ & $\begin{array}{c}\mathrm{E}_{\mathrm{u}} \\
\pm 0.01(\mathrm{eV})\end{array}$ & $\begin{array}{c}\beta \\
\times 10^{-2}\end{array}$ & $\begin{array}{c}\text { Max. Ref. } \\
\text { Index }\end{array}$ \\
\hline $195 \mathrm{~nm}$ & 3.02 & 0.66 & 3.91 & 2.14 \\
$825 \mathrm{~nm}$ & 3.01 & 0.78 & 3.30 & 2.43 \\
\hline
\end{tabular}




\section{Photoluminescence studies}

In contrast to the porphyrazines, TTBNc films show usually broad but distinct emission bands centered at the given maxima $\left(\lambda_{\text {lum }}=417 \mathrm{~nm}\right.$ and $399 \mathrm{~nm}$ for $\mathrm{t}=195 \mathrm{~nm}$ and $\lambda_{\text {lum }}=410 \mathrm{~nm}$ and $392 \mathrm{~nm}$ for $\mathrm{t}=825 \mathrm{~nm}$ ). TTBNc thin films are the first ever porphyrazines found to exhibit two luminescence peaks in the solid state ${ }^{22}$. Even though the excitation wave length gets varied to $5 \mathrm{~nm}$ from $300 \mathrm{~nm}$, there is no further shift in peaks at $392 \mathrm{~nm}(\mathrm{t}=825 \mathrm{~nm})$ and $399 \mathrm{~nm}(\mathrm{t}=195 \mathrm{~nm})$. With the increase in thickness, the intensity in emission peak get decreases from $9 \times 10^{6}(\mathrm{t}=195 \mathrm{~nm})$ to $5 \times 10^{6}(\mathrm{t}=825 \mathrm{~nm})$ at a wave length of $415 \mathrm{~nm}$ while there is a blue shift observes. This type of emission may be due to the presence of oxygen related defects, non bridged oxygen hole centers and organic bonds in form of -O-C-O ${ }^{23}$. The fluorescence spectra of naphthalocyanine with different thickness are shown in Figure 10.

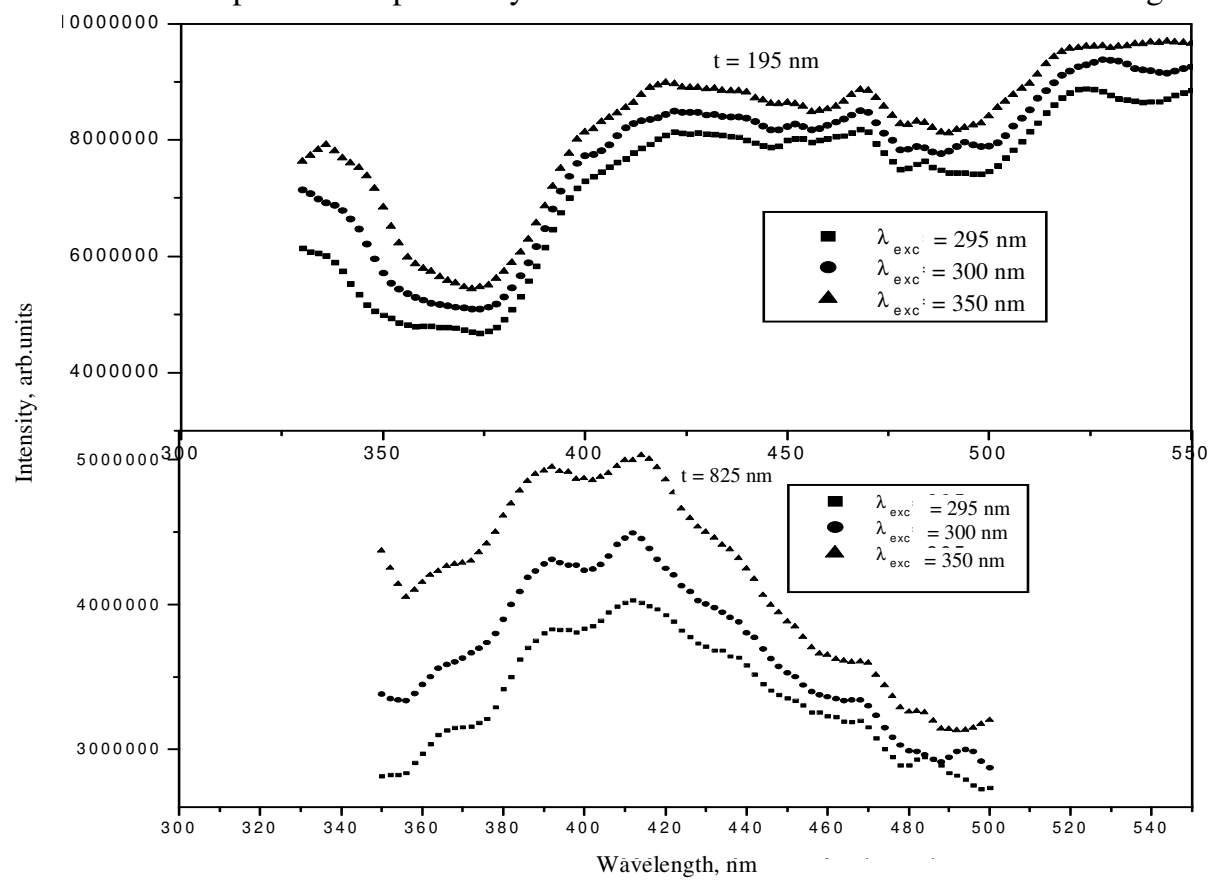

Figure 10. Luminescence spectra of TTBNc thin films as a function of wavelength for different thicknesses

\section{Conclusion}

The optical properties of amorphous tetra-tert-butyl naphthalocyanine thin films have been investigated. Using optical method, the values of different optical parameters like optical bandgap, $E_{g}$, Urbach energy, $E_{U}$ and Steepness parameter, $\beta$ are calculated. The increase in thickness scarcely changes the direct band gap, where as more deposition of material in thin film form changes the optical constants like refractive index, Urbach energy, extinction coefficient, dielectrical constants, optical conductivity and surface and volume energy losses. The fluorescence emission of TTBNc thin films shows strong broad and distinct bands with two resonance peaks. Further there is no considerable shift in peaks for single thickness film with different excitation wavelengths. As the thickness increases, it is found that there is a blue shift in the peaks. 


\section{References}

1 Leznoff C C and Lever A B P, Phthalocyanine, Properties and Applications; VCH: Newyork, 1989, 3.

2 Komori T and Amao Y, J Porphyrins Phthalocyanines, 2002, 6(3), 211-216.

3 Aranyos V, Hjelm J, Hagfeldt A and Grennberg A, J Porphyrins Phthalocyanines, 2001, 5, 609-616.

4 Turukhin A V, Gorokhovsky A A, Moser C, Solomatin I V and Psaltis D, J Luminescence, 2000, 86(3), 399-405.

$5 \quad$ Kessler B, Appl Phys A, 1998, 67, 125-133.

6 Ottaviano L, Lozzi L, Ramondo F, Picozzi P and Santucci S, J Electron Spectrosc Relat Phenom., 1999, 105, 145.

7 Biswas I, Peisert H, Schwieger T, Dini D, Hanack M, Knupfer M, Schmidt T and Chasse T, J Chem Phys., 2005, 122(6), 064710/1-064710/8.

8 Pop D, Winter B, Freyer W, Hertel I V and Widdra W, J Phys Chem B, 2003, 107, 11643.

9 Hanack M, Knecht S and Polley R, Chem Ber., 1995, 128, 929.

10 Pop D, Winter B, Frayer W, Widdra W and Hertel I V, J Phys Chem B, 2005, 109(16), 7826-7833.

11 Sakakibara Y, Bera R N, Mizutani T, Ishida K, Tokumoto M and Tani T, J Phys Chem B, 2001, 105, 1547-1553.

12 Maissel L I and Glang R, Hand Book of Thin Film Technology; Mc Graw- Hill:New York, 1983.

13 Aburaya Kamath G B, Joseph C M and Menon C S, Mater lett., 2002, 57, 730.

14 Rajesh K R and Menon C S, Mater lett., 2001, 51, 266.

15 Tauc J, Grigorovici R and Vancu A, Phys Status Solidi., 1966, 15, 627-637.

16 Tauc J, The Optical Properties of Solids; Ed., Abeles F, North Holland: Amsterdam, 1972.

17 Urbach F, Phys Rev Lett., 1953, 92, 1324.

18 Mahz H, Phys Rev., 1962,125, 1510.

19 Walaton A K and Moss T S, Proc Roy Soc A, 1963, 81, 5091.

20 Caglar Y, Ilican S and Caglar M, Eur Phys J B, 2007, 58, 251-256.

21 Ammar A H, Farid A M and Seyam M A M, Vacuum, 2002, 66, 27.

22 Frayer W, Neacsu C C and Raschke B M, J Lumin., 2008, 128, 661.

23 Fernandez T T, Jose G, Ward M, Arun Kumar K V and Unnikrishnan N V, J Appl Phys., 2009, 105, 043513. 


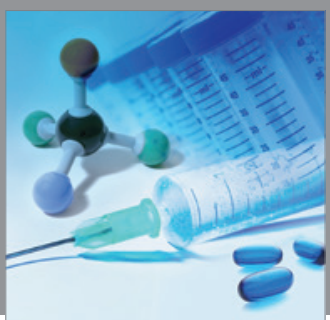

International Journal of

Medicinal Chemistry

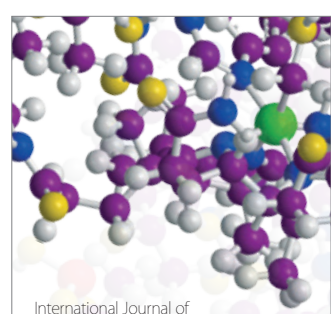

Carbohydrate Chemistry

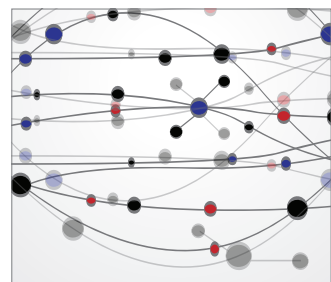

The Scientific World Journal
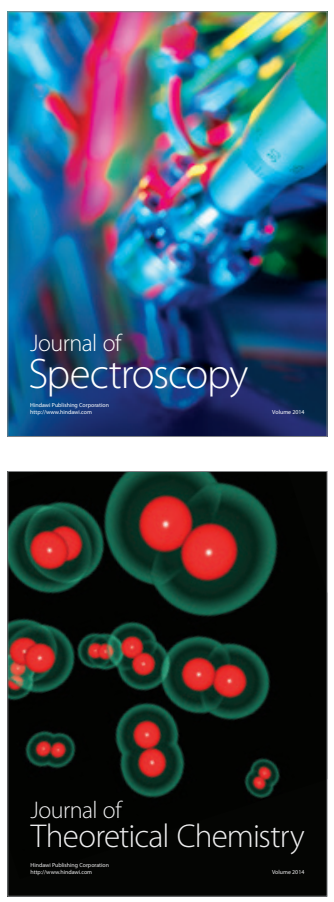
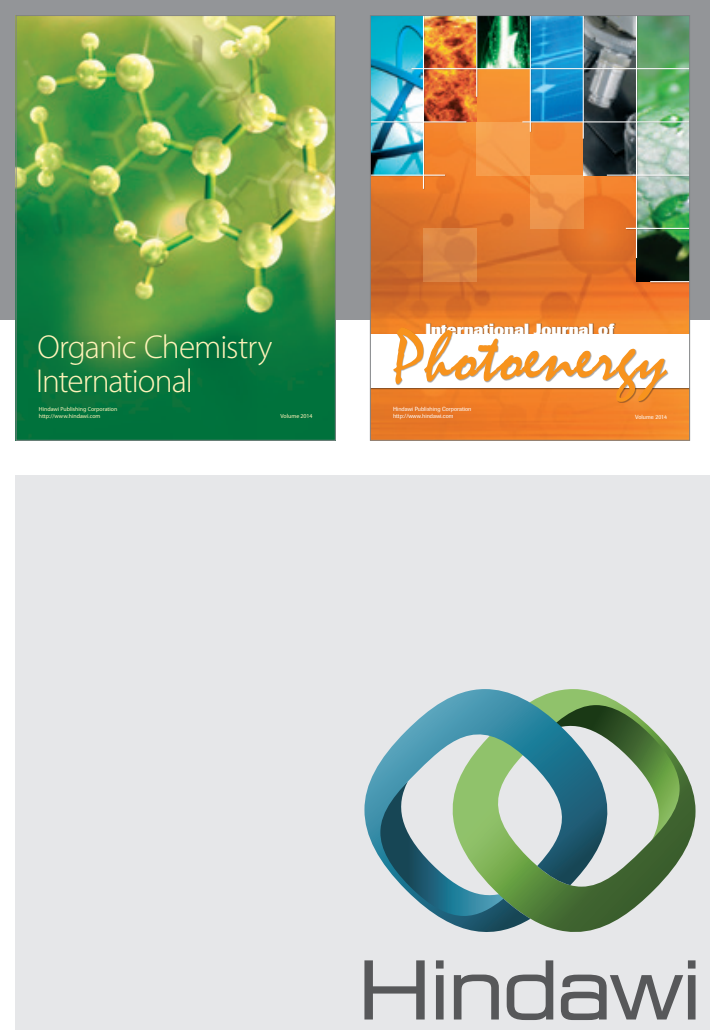

Submit your manuscripts at

http://www.hindawi.com
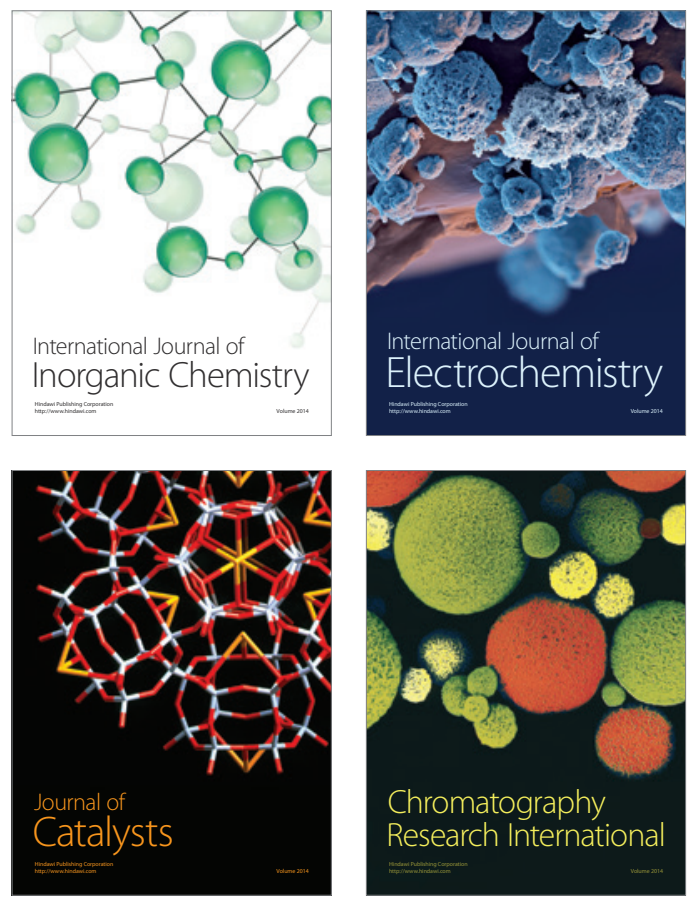
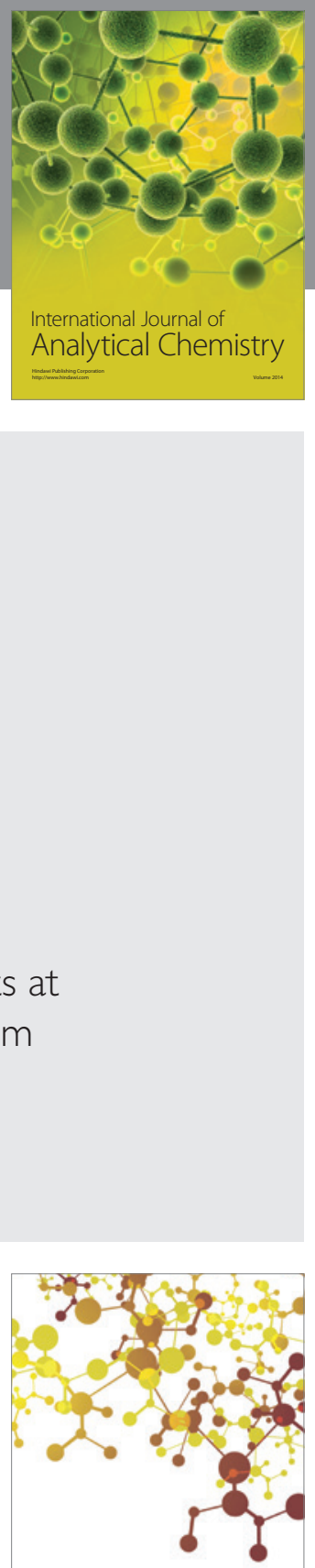

Journal of

Applied Chemistry
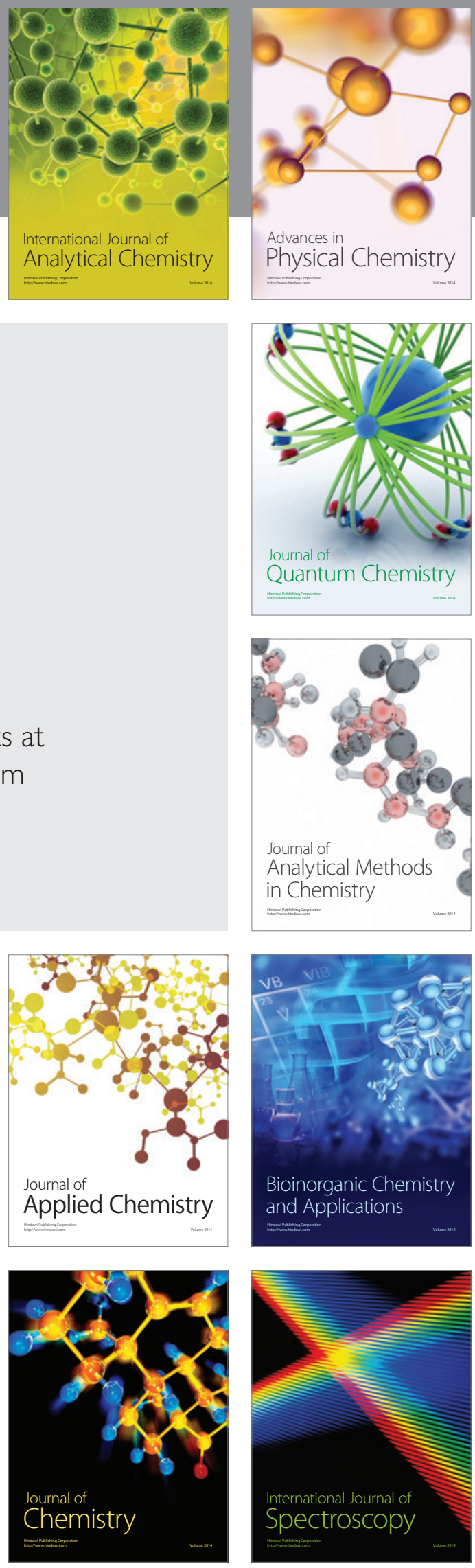